\title{
Cronologías altas, cronologías cortas: implicaciones en el debate del Paleolítico inferior Europeo
}

\author{
Juan Antonio Martos Romero*
}

el lugar en el que encontrar el centro es en el límite ese repiqueteo en el cielo no es más que una cañería que vibra (Bukowski)

\section{RESUMEN:}

En el presente artículo se revisan los argumentos y premisas asumidos por los diferentes investigadores en torno a las controversias que para el primer poblamiento de Europa ha suscitado la polémica entre las cronologías altas y cortas y sus implicaciones en el momento actual de revisión del Paleolítico inferior europeo. Pensamos que las cuestiones cronológicas solamente puede ser debatidas con validez partiendo de un contexto geográfico que supere el esencialmente europeo. Desde esta perspectiva las cronologias altas se han mostrado hasta ahora incapaces de generar un marco teórico alternativo para explicar una secuencia industrial pliopleistocena construida esencialmente con argumentos tipológicos y extrapolada de la africana con serias dicotomías cronológicas, hoy por hoy, lejos de solucionar. Por último, se comenta el hallazgo en los útlimos años de yacimientos en la Península lbérica con fechas cercanas al millón de años que vienen a plantear nuevos e interesantes interrogantes en este debate.

- Becario Predoctoral del Departamento de Prehistoria e H. ${ }^{a}$ Antigüa de la Universidad Nacional de Educación a Distancia (UNED). C/ Senda del Rey s/n., 28040 Madrid. 


\section{ABSTRACT:}

In this paper are revised the arguments assumed by the different scholars in the discussion about the first settlement of Europe with the controversy arised between the called "long chronology" and "short chronology", and its implications in the debate of the Lower Paleolithic of Europe. In our opinion, chronological questions only can be discussed from a geographical context that includes Africa and the Next Orient. From this perspective the "long chronology" seems unable to create a theoretical framework to explain an industrial sequence plio-pleistocene made exclusively whit typological arguments and extrapolated from the African sequence with clear chronological dichotomies. Finally, we want to denote the finding in the last years of several sites in the lberian Peninsula with dates around 1 myr ago that introduce new and interesting aspects in this debate.

En los últimos años hemos asistido a una clara e interesante polémica en torno a la cuestión de la cronología del primer poblamiento de Europa con el resultado de una polarización de los investigadores en dos posturas bien diferenciadas. En el presente artículo no pretendo realizar una revisión crítica de las evidencias arqueológicas empleadas, bien por áquellos que postulan un poblamiento temprano que incluso hacen remontar a los límites del Plio-Pleistoceno, o bien por quienes defienden que dicho poblamiento, o al menos ocupación efectiva del continente, no puede llevarse más allá de los límites del Pleistoceno Medio y en la práctica a la fecha de los 500.000 años B.P ${ }^{1}$.

Por contra, intentaré exponer de forma sucinta los paradigmas sobre los que se sostienen ambas posiciones, las premisas que asumen y por último las implicaciones que ha provocado en la discusión del Paleolítico Inferior europeo el debate de las cronologías altas o cortas en el poblamiento de Europa.

1 Existen un buen número de publicaciones en las que se realiza una revisión más o menos crítica de las evidencias arqueológicas y paleoantropológicas que han servido de base para la aparición de las cronologías altas y que contemplan esta problemática desde ambas posiciones;

a) Desde la órbita de los investigadores que apoyan las cronologias altas pueden consultarse, VALOCH (1976a) o BonIFAY y VANDERMEERSCH (1991a).

b) Desde la perspectiva crítica de las cronologías cortas destacan los artículos de ROEBROEKS y KolfsChOten (1994 y 1995a y b). Para trabajos centrados en las evidencias de la Península Ibérica, Raposo y SANTONJA (1995). 


\section{LA «REVOLUCIÓN"DE LAS CRONOLOGIASS ALTAS}

En el IX Congreso de la UISPP que tuvo lugar en Niza en 1976 se planteó por primera vez de forma explícita una cronología alta para el poblamiento de Europa. En el volumen que recogía las ponencias del coloquio VIII bajo el título de Les premiéres industries de l'Europe (Valoch, 1976a) se dieron a conocer nuevas industrias repartidas fundamentalmente por el litoral mediterráneo y la región centroeuropea a las que se atribuían fechas muy altas del Pleistoceno Inferior ${ }^{2}$ (figura 1).

Sin embargo, los sitios e industrias que habian sido presentados entonces como claras evidencias de ese temprano poblamiento se vieron sometidos pronto a fuertes controversias y críticas. La inexistencia de buenos conjuntos líticos, en numerosas ocasiones compuestos tan sólo por escasas piezas recogidas en lugares sin contextos estratigráficos bien definidos, donde por otra parte resultaba enormemente difícil diferenciar trazas de una autoría humana en su realización (hecho que se explicaba como consecuencia de unas tecnologías "primitivas" que daban como resultado tipologías poco varíadas y de formas también "primitivas" en buena consonacia con la concepción de la evolución gradual de las industrias del paleolítico entonces imperante), junto a la ya comentada falta de rigor en la documentación de los contextos estratigráficos y con ello la escasa objetividad de las dataciones propuestas; llevó, con algunos años de diferencia, a la realización de dos nuevas reuniones centradas en esta cuestión. Si bien ambas estuvieron motivadas por una misma intención, ante la práctica ausencia de una discusión científica en torno al asunto del comienzo del poblamiento de Europa, las conclusiones alcanzadas han sido muy diferentes y han dado lugar a la ya señalada polarización de posiciones. Estoy haciendo referencia al denominado congreso de París celebrado en 1989 (Bonifay y Vandermeersch, 1991a) y al workshop de Tautavel (Francia) en 1993 (Roebroeks y Kolfschoten, 1995a).

El congreso de París (1989) acabaría refrendando y ampliando las conclusiones de Niza (1976) dando su apoyo a las cronologías altas. Sin haber solucionado de modo satisfactorio, en mi opinión, las carencias cien-

2 "Nous pouvons donc, clonclure notre colloque par la constatation que, d'aprés les résultats des récherches les plus récentes, les gens firent leur apparition dans la partie méditerranéenne de l'Europe il y a peut-être déjà un million cinq cents mille ans; de l'époque d'il y a environ un millon jusque 700 mille ans, nous connaisons dejà toute une suite d'habitats se trouvant dans l'Europe Méditerranéenne et Centrale. En conclusant, j'ose exprimer ma conviction que les fouilles et les études qui seront réalisées á l'avenir feront reculer cette limite chronologique du peuplement de l'Europe encore plus avant dans le passé." (VALOCH, 1976b, pp. 183). 


\title{
UNION INTERNATIONALE DES SCIENCES PRÉHISTORIQUES ET PROTOHISTORIQUES
}

Membre du Conseil International de la Philosophie ef des Sciences Humaines de I'UNESCO, PARIS

\section{IX' \\ CONGRÈS}

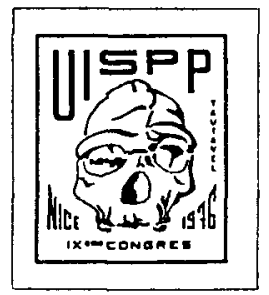

COLLOQUE VIII

\section{LES PREMIËRES INDUSTRIES \\ DE L'EUROPE}

\author{
PRÉTIRAGE \\ Direction \\ Karel VALOCH \\ Moravske Museum, Brno, Tchécoslovaquie \\ NICE \\ Parc Valrose de l'Université de Nice \\ 13-18 Septembre 1976
}

Figura 1. Portada del VIII Coloquio del IX Congreso de la UISPP (Niza, 1976). 
tíficas de que adolecían las evidencias presentadas en cuestiones tan importantes como los contextos geológicos y estratigráficos o la datación objetiva de los mismos se planteó lo que podemos denominar desde el punto de vista diacrónico una pequeña "revolución". La ampliación del marco cronológico del Paleolítico Inferior europeo hasta fechas equiparables a las obtenidas en el continente africano suponía, a juicio de los investigadores que defendian la existencia de industrias pliopleistocenas europeas, una innovación en la investigación que obligaba a replantearse y renovar los presupuestos teóricos que hasta aquel momento habian sido utilizados para construir el paradigma de la expansión humana desde África y su llegada a Europa ${ }^{3}$.

Este afán de superación de esquemas teóricos que supuestamente estarían impidiendo el avance de nuevas investigaciones esconde, tal y como algunos autores han acertado a ver (Santonja, 1995), una posición anclada en el paradigma francés construido por Bordes entre los años 50 y 70 sobre la secuencia y evolución de las industrias del paleolítico inferior europeo y que desde mediados de esa última decada se encontraba en revisión. Bordes estableció una sucesión de estadios extraída esencialmente de las terrazas del valle del Somme (Francia) y puesta en consonacia con el cuadro cronoclimático del modelo de las glaciaciones alpinas por entonces asumido ${ }^{4}$;

\begin{tabular}{|c|c|}
\hline \multicolumn{1}{|c|}{ INDUSTRIAS } & GLACIACIÓN \\
\hline Abbevillense & Mindel \\
\hline Achelense; & Mindel-Riss / Würm I \\
& Anterior al Riss I \\
& A partir del Riss I \\
& Medio \\
& Superior \\
\hline
\end{tabular}

3 "Pour résoudre, au cours des années á venir, l'ensemble des problémes concernant l'origine des peuplements européens, dans le cadre de l'evolution génerales des Hominidés, les chercheurs devront faire table rase d'un certain nombre d'hypothéses actuelles et d'idées reçues qui bloquent la recherche en considérant qu'un fait ne peut être vrai... "parce qu'il n'est pas possible"! Pour faire avancer ces recherches il faudra innover, accepter ce qui peut paraître aujourd'hui invraisemblable si cela s'avère vrai, étayé par des faits indiscutables." (BONIFAY y VANDERMEERSCH, 1991b, pp. 8).

4 Pronto pudo comprobarse que la secuencia se mostraba incompleta;

"...puesto que durante las fases frías la erosion sometería a intensos procesos de denu. dación las laderas de los valles, y las industrias de los interglaciares habrian, en gran parte desaparecido. Asi se explicaba que el Achelense medio (...), fuera el mejor representado en el norte de Francia." (SANTONJA, 1995, pp. 54). 
Para aquellas industrias anteriores al Würm I que se salian de los tipos registrados en la secuencia clásica de las terrazas del Somme se creó una denominación de facies regional, el "Achelense meridional" (Villa, 1981) 5 .

En resumen, el paradigma establecido por Bordes (Bordes, 1971) planteaba una evolución diacrónica de las industrias, de signo gradualista, hacia formas más simétricas o típicas y menos «primitivas», explicándose las diferencias regionales por una diversificación supuestamente sincrónica del achelense en facies geográficas que traducian la existencia de estilos regionales distintivos (Villa, 1981). Un marco teórico como el expuesto, donde los estadios evolutivos quedaban comprendidos en un margen cronológico relativamente escaso debido a que las variaciones tipológicas tenían que ajustarse al modelo climático previamente asumido, sólo podía explicarse si se admitía que se habían dado transformaciones rápidas en las industrias. Este hecho resultaba evidentemente problemático desde la propia perspectiva gradualista del esquema de Bordes dado el claro contraste que existía entre el escaso lapso temporal concedido a esta secuencia y la asumida complejidad de las industrias del paleolítico inferior europeo (Santonja, 1995).

La pretentida sucesion de rápidas transformaciones en las industrias achelenses europeas también parecía contradecirse con la estabilidad tipológica y tecnológica que reflejaban las industrias achelenses en África sin cambios significativos prácticamente a lo largo de todo el Pleistoceno Inferior y Medio.

Villa (1981) subrayó que frente a este esquema de facies regionales y de rápidas transformaciones en las industrias, en realidad el Pleistoceno Medio parece responder más bien a un periodo uniforme, donde la imagen de estabilidad en las industrias se muestra mucho más real que la ilusión de diferenciaciones regionales 0 de progresiones de carácter temporal en los conjuntos líticos ${ }^{6}$. En primer lugar porque no estaría demostrada la "contemporaneidad" de los conjuntos que sostienen la idea de las distintas facies regionales y en segundo porque las variaciones tipológicas se

5 Tal y como han reseñado algunos investigadores (SANTONJA, 1995; VILLA, 1981), esta denominación de facies regional llegó a extrapolarse a algunos conjuntos de la Península lbérica como los de Torralba con diferentes variantes hasta finalmente plantearse la existencia de contactos con África (Alimen, 1975; Bordes y THIBAULT, 1977)

- "En una escala amplia se constata claramente que pese a las diferencias existe una alto grado de universalidad y homogeneidad en las industrias del paleolitico inferior. No existe pues una correlación clara entre la diversidad de medios y la que presentan las industrias liticas, esa diversidad de medios no se refleja. Cierto es que como ya hemos dicho, resulta sumamente complejo realizar comparaciones a nivel regional porque faltan dataciones absolutas que permitan establecer sincronias. No obstante, esa homogeneidad en las industrias puede que esté reflejando una escasa capacidad de adaptación a diferentes medios, como ha señalado Villa (1991)." (MARTOS, 1994, pp. 38). 
explican mucho mejor en función de la utilización de materias primas diferentes y de disponibilidad local como ya desde un principio defendieron algunos investigadores ${ }^{7}$. Este factor tendría, al menos, un importante peso en una tecnología simple como la de estas industrias. En todo caso antes que plantearse posibles afinidades culturales o la existencia de tradiciones regionales, parece más lógico buscar la causa de dichas variaciones en factores como el tiempo, el espacio o incluso la función, uso o habilidad del autor (Villa, 1981; Raposo y Santonja, 1995).

El debate entre quienes mantenían las diferenciaciones geográficas y áquellos que se oponían a contemplar las diferencias en los conjuntos achelenses como reflejo de una diversificación en facies regionales se centró en una distinta concepción de las formas de vida y de la organización social de los grupos del Pleistoceno Medio; estructuras sociales cerradas con tradiciones regionales frente a pequeños grupos, relativamente móviles, y ampliamente dispersos en redes abiertas (Villa, 1981).

Partiendo de este marco de discusión, podemos afirmar que el congreso de París supuso un refuerzo para el paradigma francés de evolución gradual de las industrias, como consecuencia de la creación de un cuadro cronológico de gran antigüedad para el Paleolítico Inferior europeo con fechas similares a África. En realidad este cuadro había sido construido con argumentos exclusivamente tipológicos (puesto que la problemática surgida en torno a la entidad de estas industrias no fue solucionada en su totalidad), basado en la existencia de industrias sin bifaces con cronologias del Plio-Pleistoceno y del Pleistoceno Inferior que eran el resultado de la extrapolación de la secuencia africana creándose un paleolítico arcaico en Europa, el Trés Ancien Paleolithique (figura 2). La secuencia estaba basada en escasas y diseminadas industrias desarrolladas supuestamente entre 2'5 y 0'6 m.a. (Bonifay y Vandermeersch, 1991b, pp. 315);

\begin{tabular}{|c|c|}
\hline INDUSTRIAS & CRONOLOGIA \\
\hline $\begin{array}{c}\text { Fase arcaica del «Trés Ancien } \\
\text { Paléolithique» }\end{array}$ & 2,5 a 1 m.a. \\
\hline $\begin{array}{c}\text { Fase evolucionada del «Trés Ancien } \\
\text { Paléolithique» } \\
\text {-Abbevillense- } \\
\text {-Achelense antiguo- } \\
\text {-Achelense- }\end{array}$ & 1 a 0,6 m.a. \\
\hline
\end{tabular}

7 Jelinek $(1975,1976)$; Tavoso $(1975,1978)$; Jones (1979); Straus (1980) y FiSH (1981). 


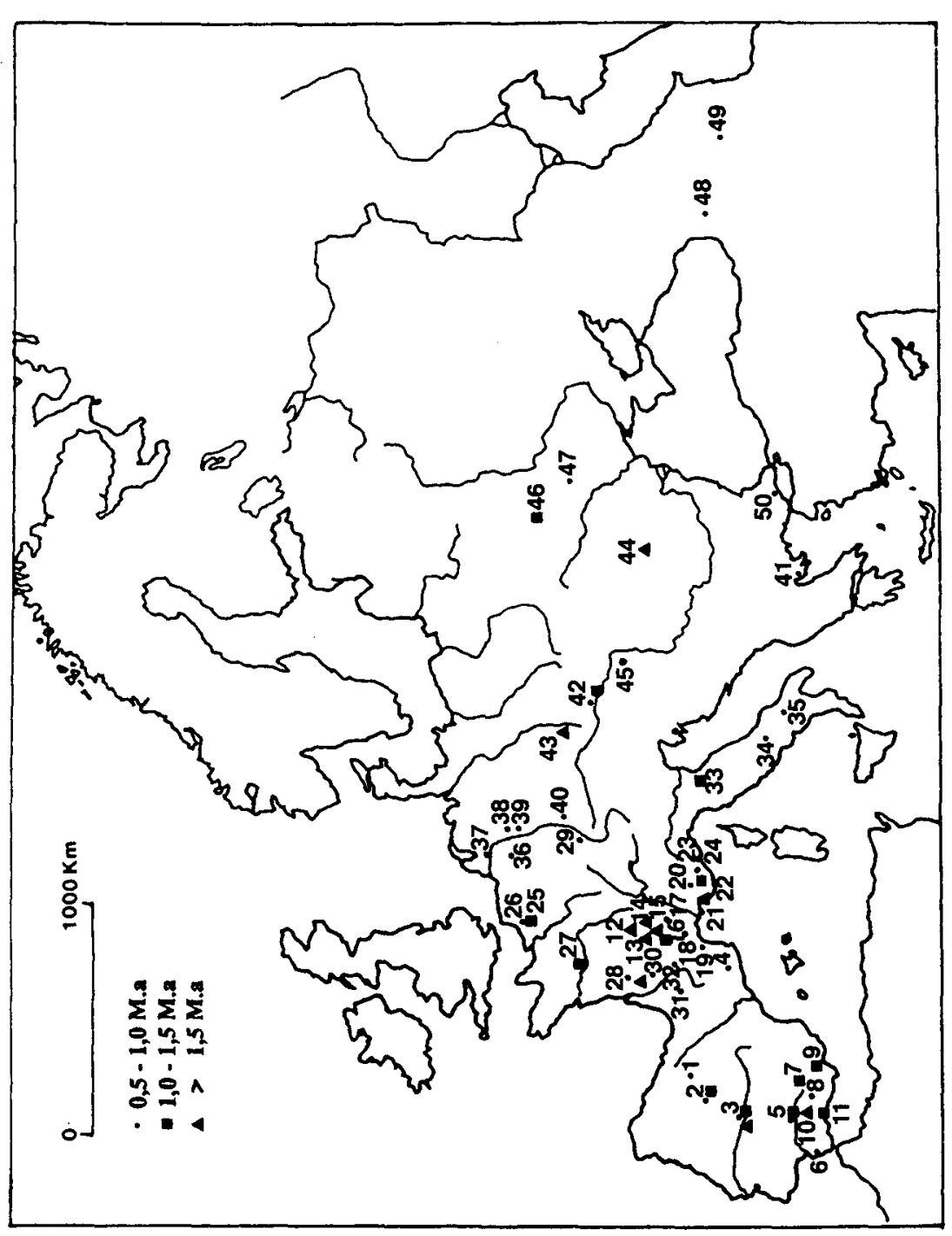

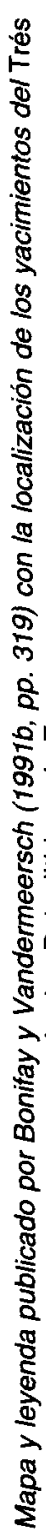

พัฒ 
1. Atapuerca (Espagne)

2. VALLEE DU DUERO (Espagne)

3. ValLeE du TAJo (Espagne)

4. ValleE dU Ter (Espagne)

5. ValleE du Guadalouivir (Espagne)

6. CÁdiz-Aculadero (Espagne)

7. Guadalete (Espagne)

8. Cortillo de Dona Millagros (Espagne)

9. CUEVA VICTORIA (Espagne)

10. CoRTILlo de DON AlFonso (Espagne)

11. OrCE (Espagne)

12. Perier-Etoualres (France)

13. Saint-Eble; Le Coupet (France)

14. CHILHAC; BLASSAC (France)

15. LA ROCHELAMBERT (France)

16. NOLHAC; SAinzelles (France)

17. SoleiHAC (France)

18. GRotte d'Aldene (France)

19. Grotte de TAutavel (France)

20. Grotte de L'EsCALE (France)

21. MARSEILle (France)

22. BESSE SUR ISSOLE (France)

23. VIDAuban (France)

24. Grotte du Vallonnet (France)

25. MONTIERES (France)

26. ABbeVILLE (France)

27. LA HULANDERIE (France)

28. Grotte d'ARTENAC (France)

29. ACHENHEIM; HaNGenbieten (France)

30. BASSIN DE L'ISLE (France)

31. Grotte de LaRomieu (France)

32. GROTTE VAUfREY (France)

33. MONTE POGgIOLO (Italie)

34. ISERNIA (Italie)

35. VENOSA (Italie)

36. Grotte de Belleroche (Belgique)

37. RHENEN (Pays-Bas)

38. KaRLICH (Allemagne)

39. MISENHEIM (Allemagne)

40. Mauer (Allemagne)

41. Petralona (Grèce)

42. Stranska-Skala (Tchécoslovaquie)

43. BEROUN (Tchécoslovaquie)

44. Bassin Dacioue (Romanie)

45. VERTESSOLLOS (Hongrie)

46. KOROLEVO (URSS)

47. LUKa-VRUBLEVETSKAJA (URSS)

48. DMANICI (URSS)

49. AZICH (URSS)

50. Yarimburgaz (Turquie) 
Los problemas que se habian puesto de manifiesto para la secuencia establecida por Bordes, son también aplicables a los estadios evolutivos del Trés Ancien Paleolithique desde la estabilidad de las industrias achelenses del África Oriental. Un marco como el propuesto por las cronologias altas se ve obligado a asumir un planteamiento de claro corte gradualista en la evolución de las industrias europeas basado fundamentalmente, como ya he señalado, en argumentos tipológicos extrapolados de la secuencia africana que, sin embargo, introduce serios desfases cronológicos con ésta última y que chocan frontalmente con la estabilidad que reflejan las industrias achelenses africanas. En este continente las primeras industrias achelenses aparecen en torno a 1,7/1,6 m.a., no alcanzando su pleno desarrollo o estandarización hasta hace 1,5/1,4 m.a. (Roche, 1996), y no se introducen cambios significativos, es decir, permanecen estables hasta $0,7 / 0,4$ m.a. Si volvemos la mirada a Europa y la secuencia de industrias propuesta por las cronologías altas comprobamos que resulta díficil justificar esta extrapolación con un desfase cronológico de prácticamente un millón de años en el paso de las industrias sin bifaces (olduvaienses) a las achelenses y de otros 300.000 a 500.000 años para la estandarización de ese utillaje bifacial. A lo que se añade el hecho de que este último paso habria sido llevado a cabo por protagonistas diferentes, Homo erectus en África y Homo sapiens neandertalensis en Europa (Santonja, 1995).

\section{LA PERSPECTIVA CRÍTICA DE LA CRONOLOGIA BAJA}

La revisión crítica acerca de la entidad de las evidencias arqueológicas y paleoantropológicas manejadas por los defensores de una cronología alta para el poblamiento de Europa que se realizó en la reunión de Tautavel (1993) abrió la visión escéptica que en torno a esta cuestión mantienen en la actualidad los investigadores que proponen una cronología corta para dicho poblamiento. El enfoque del problema desde un contexto geográfico mucho más amplio puso de manifiesto las carencias teóricas que las cronologías altas habian mostrado para generar desde sus planteamientos un paradigma alternativo al hasta ahora mantenido para explicar la expansión de los homínidos desde África.

Se acusa a los investigadores partidarios de las cronologías cortas de negarse a aceptar cualquier evidencia que supere la fecha de 0,5 m.a. simplemente porque tal situación se escapa a los esquemas teóricos asumidos (Peretto, 1996). Lo que se plantea, por contra, es la necesidad de analizar estos conjuntos bajo unas mínimas expectativas científicas con dos puntos esenciales (Roebroeks y Kolfschoten, 1994); 
a) un profundo estudio de toda la gama de condiciones naturales que rodean el sitio y que pudieran haber producido formas semejantes a las de los artefactos realizados por homínidos.

b) estudio minucioso de las características geológicas y geomorfológicas de los sitios donde las piezas líticas son recogidas.

Sólo cumpliendo estos requisitos se puede trabajar con evidencias firmes que permitan elaborar nuevos interrogantes e hipótesis.

El planteamiento de las cronologías cortas para el poblamiento del continente europeo admite la posibilidad de una presencia de grupos aislados en Europa con fechas anteriores a los 500.000 años B.P., cuestión a la que posteriormente volveremos a prestar atención, pero sitúa en ese momento la ocupación efectiva del continente (Roebroeks y Kolfschoten, 1994; 1995b). Los investigadores que se afirman en esta postura han señalado que desde esa fecha se puede comprobar la existencia de industrias bifaciales evolucionadas en yacimientos como Boxgrove (Reino Unido) (Roberts, 1990; Roberts et al, 1994; Gamble, 1994), hecho que encaja bastante bien con las industrias del tipo achelense superior que aparecen en África en torno a $0,7 \mathrm{~m}$.a. La estabilidad de las industrias achelenses en África se explica mucho mejor desde esta perspectiva de poblamiento tardío de Europa y de monotonía del Pleistoceno Medio europeo frente a la imagen de rápidas transformaciones ${ }^{8}$.

De la misma manera se logra superar el paradigma de Bordes ya que la existencia desde muy pronto en Europa de industrias bifaciales evolucionadas obliga a desechar la división del Paleolítico inferior europeo en estadios desde la óptica de una concepción evolutiva de las industrias basada en argumentos tipológicos hoy por hoy descartados como marcadores válidos de sucesión en el tiempo; industrias arcaicas, abbevillenses, achelense inferior, medio y finalmente superior, construida con pobres y escasos conjuntos a la vez que sin sólidas referencias cronológicas (Raposo y Santonja, 1995).

8 ... si tenemos en cuenta que desde el estadio isotópico 7 comienzan a registrarse industrias diferentes, más complejas tanto desde el punto de vista tecnológico como conceptual, encuadrables en el Paleolítico Medio, el achelense (...) se reduciria prácticamente a los conjuntos industriales entre los estadios isotópicos 13 y 8 , siendo además los anteriores al 9 más bien escasos, 10 que encaja perfectamente con la estabilidad que observamos en estas industrias, muy homógeneas cuando circunstancias como medio ambiente y materias primas coinciden." (SANTONJA, 1995, pp. 59). 
Raposo y Santonja (1995) dejan ver en este sentido que el caso de la Península lbérica es bien significativo. Los conjuntos que están formados por amplias series industriales ya contienen bifaces con lo que la existencia de un posible estadio preachelense identificado en algunos yacimientos, con mayor o menor fortuna, debe tratarse con mucha precaución. Estos autores han llevado a cabo una exhaustiva y completa revisión crítica de tales yacimientos. Entre los citados quiero destacar dos yacimientos por las revisiones de que han sido objeto, El Aculadero en Cádiz (Querol y Santonja, 1983) y Pinedo en Toledo (Querol y Santonja, 1979), demostrando que los argumentos tipológicos carecen de valor cronológico. Su conclusión es que, a falta de trabajos centrados en la datación de sitios arqueológicos o de formaciones geológicas en medios fluviales, como los emprendidos en las terrazas del Guadalquivir y del Tajo, el inicio de la presencia humana en la Península lbérica debe datarse en torno al comienzo del Pleistoceno Medio y que en función de los datos actuales no es posible distinguir ni la presencia de fases preachelenses ni de estadios Achelenses primitivos ya que todas las industrias bifaciales se encuadran en un Achelense pleno (Raposo y Santonja, 1995) ${ }^{9}$.

La posibilidad de una ocupación anterior de carácter intermitente que parece verse reforzada con yacimientos como T6 y T8 en el Guadalquivir (Díaz del Olmo et al, 1993) o Cúllar Baza I en Granada (Ruiz Bustos y Michaux, 1976) y fundamentalmente Fuentenueva 3 en la región de Orce en Granada ${ }^{10}$ y Atapuerca TD6 en Burgos (Parés y Pérez-González, 1995; Carbonell et al, 1995) con dataciones que podrían colocarse por encima del evento Jaramillo, abre la cuestión de un poblamiento de la Península Ibérica en el Pleistoceno Inferior hacia $0,8 / 0,9$ m.a. La presencia de estos grupos aislados en el límite entre el Pleistoceno inferior y el medio podría entonces explicarse como fruto de un poblamiento esporádico de las regiones europeas más favorables en términos de subsistencia hasta su ocupación efectiva hace 500.000 años con un origen común en Homo erectus y el achelense de África Oriental y que con posterioridad pudieron diversificarse (Santonja, 1995) (figura 3).

En todo caso lo que las cronologías cortas han denunciado es la necesidad de contemplar estos datos y la problemática implicada desde un

9 «In relation to the Middle Pleistocene phases, the sole aspect to be retained is the scarcity of bifaces prior to OIS 9 and their abundance thereafter..." (RAPOSO y SANTONJA, 1995, pp. 21).

${ }_{10}$ Si bien no hay en la actualidad publicaciones detalladas sobre este yacimiento, el sitio, excavado por Alain Turcq, fue presentado a la comunidad científica en el Congreso Internacional de Paleontología Humana celebrado en Orce (Granada) en el mes de septiembre de 1995. 


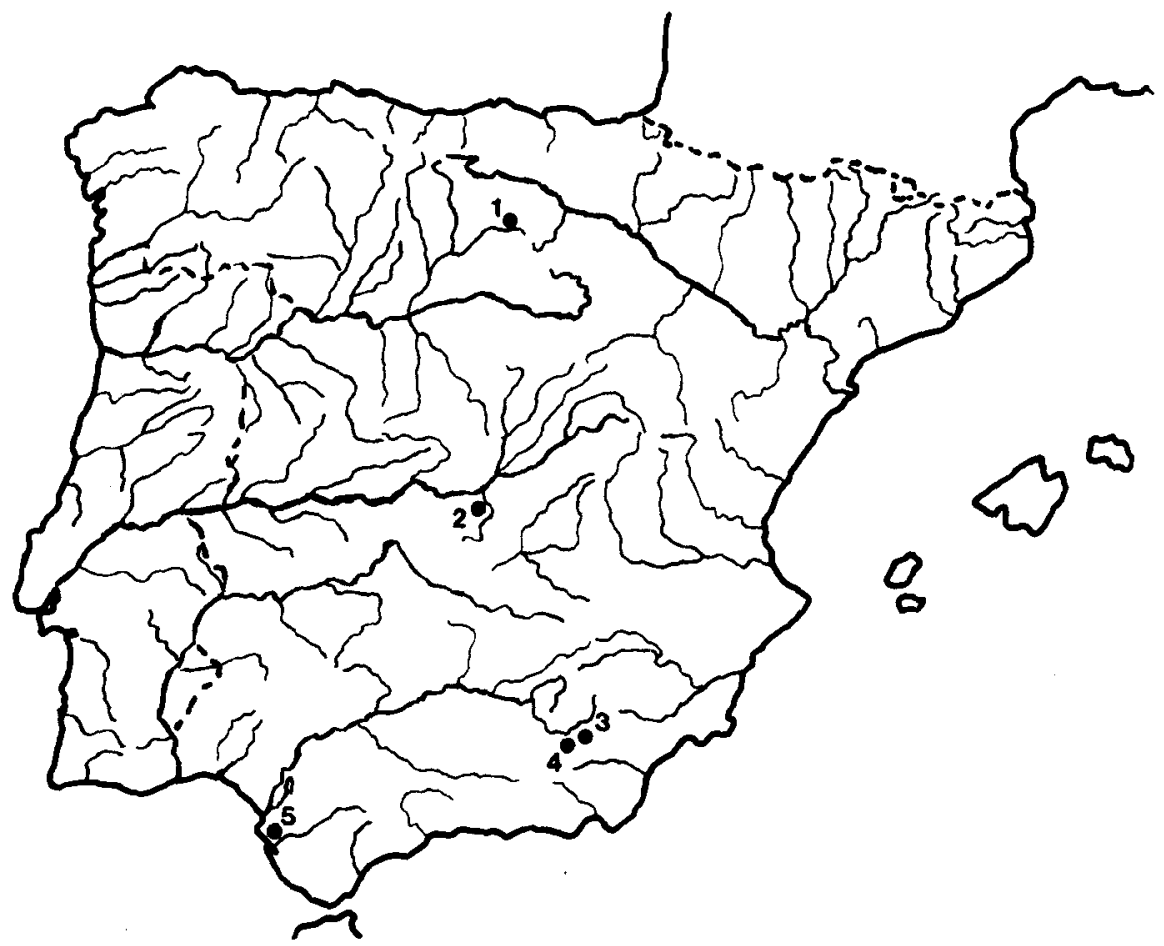

Figura 3. Localización de algunos de los yacimientos de la Península lbérica citados en el texto; 1. Atapuerca, 2. Pinedo, 3. Cúllar de Baza I, 4. Fuentenueva 3, 5. El Aculadero.

contexto geográfico significativo; África Oriental, litoral Mediterráneo y Próximo Oriente $"$.

Desde esta premisa puede comprobarse como un poblamiento de carácter intermitente $u$ ocupación esporádica incial de Europa no podría remontarse por encima de 1,7 m.a. según el paradigma actual que obliga a establecer una barrera infranqueable en la aparición de Homo erectus en África coincidiendo con las primeras industrias achelenses. Las cronologías altas no han sido capaces de poner sobre la mesa de debate un paradigma alternativo que explique la existencia de industrias en Europa en fechas anteriores a la aparición de Homo erectus y de las industrias ache-

11 "...especially because it seems clear now that around 1 Myr ago humans were already at the southern frontiers of Europe, from the Caucasian area to the littoral of Moroco." (RAposo y SANTONJA, 1995, pp. 21). 
lenses en África puesto que se verían forzados a demostrar bien (Santonja, 1995);

a) que el origen de la especie erectus es anterior a esa fecha

b) que otro homínido es el responsable de la expansión inicial desde África

c) o que no hay un sólo foco territorial en el origen del ser humano.

No obstante, como ha indicado Roche, la posible salida de África de un homínido antes de estas fechas puede plantearse como una hipótesis que sin embargo hoy por hoy está muy lejos de demostrarse (Roche, 1996).

El panorama dibujado por los datos procedentes de Asia (Swisher et al, 1994), o yacimientos como Ubeidiya en Israel, Dmanisi en el Caucaso, al igual que en norte de África Ain Hanech o Sidi Abderrahman, parece indicar que el poblamiento de Europa fue marginal desde el punto de vista diacrónico, que los grupos humanos se hallaban ya a las upuertas de Europa» en torno a 1 m.a. y que no rompieron esa barrera hasta hace 500.000 años. Se produce entonces un poblamiento veloz y prácticamente de carácter sincrónico en todas las regiones del continente en donde aparecen industrias de este momento que obliga a preguntarse qué barreras, si es que las hubo, existían antes de esa fecha, y si así fue y como consecuencia de ello qué factores climáticos y sociales hicieron posible el franquearlas. Entran en consideración aquí elementos y cuestiones en torno a la tecnología, las estrategías de subsistencia, la organización social o las capacidades neurofisiológicas (lenguaje), entre otros, de estos grupos pleistocenos (Roebroeks y Kolfschoten, 1994; 1995b).

Por contra cabe la posibilidad de que, dada la baja resolución cronológica en las dataciones manejadas para este periodo, la impresión de un poblamiento y adaptación rápida con estallidos contenidos o puntuados frente a otro progresivo desde una salida gradual y contínua de África, sea más artificial que real.

\section{REFLEXIÓN FINAL}

El principal problema con el que se han de enfretar los investigadores que defienden y apoyan una cronología alta para el poblamiento de Europa es la falta o inexistencia de un marco teórico y referencial válido en el que colocar y explicar las dicotomías cronológicas de las industrias eu- 
ropeas supuestamente pliopleistocenas con la secuencia africana, a la vez que capaz de mostrarse como una alternativa eficaz al paradigma actual para la salida de los homínidos de África ${ }^{12}$.

La aparición en los últimos años de yacimientos en buenos contextos con fechas en el millón de años abre una cuestión sumamente interesante acerca de la posibilidad de ocupaciones intermitentes en regiones que pudieran, por sus caracterísiticas ecológicas, haber permitido un poblamiento esporádico hasta el «estallido» de hace 0,5 m.a. Este pudo haber sido el caso de la Península Ibérica. Turner propone que en esta última pudo darse un poblamiento esporádico a lo largo del Pleistoceno Inferior debido a que sus especiales condiciones paleoecológicas así lo habrían posibilitado, centrando su argumentación en la composición de la fauna y fundamentalmente de la cadena trófica a partir de elementos como la estructura del grupo de grandes carnívoros y de la existencia de un menor número de especies de hyena (Turner, 1995).

Una propuesta de tales características no entra en contradicción con los planteamientos de las cronologias cortas que, desde su perspectiva crítica y abierta a los interrogantes surgidos de nuevas evidencias mínimamente objetivas, han demostrado que la problemática aquí tratada debe contemplarse desde un marco geográfico amplio que permita obtener;

“...explicaciones más universales a los mecanismos y las formas de adaptación del hombre pleistoceno, y a la vez -aunque pueda parecer una paradoja- buscar en los elementos locales particulares de cada sitio la explicación de las diferencias.» (Santonja, 1995, pp. 59).

12 Muchos de los investigadores alineados en esta postura critican la rigidez del paradigma actual, que a su juicio impide el avance de la investigación limitándose a negar aquellas evidencias que se salen del marco teorico establecido. Sin embargo, estos mismos investigadores se muestran por el momento incapaces de presentar un paradigma alternativo concentrando sus esfuerzos en la discusión acerca de la necesidad de afinar las dataciones y mejorar los métodos de datación;

"It is surprising that the first human groups that inhabited the Italian peninsula had an industry which in some aspects is similar to the oldest ones found in Africa, previous to the appearance of the Acheulean. The latest studies, which are still in course, could therefore lead to the identification of a sort of sequence of typological phenomena which in a certain sense can be superimposed with that found in Africa, but at the same time with a substantial difference. The Italian and European sequence, in fact, is currently younger by many hundreds of thousands of years. If all this is true, then it is necessary to resolve this important chronological dichotomy. Personally I belive that it is not possible to resolve it by concentrating on cultural or paleoethnological aspects but rather by insisting on greater discussion of the age of the sites and on the dating methods used, both relative and absolute." (PERETTO, 1996, pp. 15). 


\section{BIBLIOGRAFÍA}

Aguirre, E., (1996): “Orígenes del Poblamiento en la Península lbérica». En El Hombre Fósil 80 años después; A. Moure Romanillo (ed.), Homenaje a Hugo Obermaier. Universidad de Cantabria, pp. 127-151.

ALIMEN, H., (1975): "Les isthmes hispano-marocain et sicilo-tunisien aux temps acheuléens." L'Anthropologie, 79, pp. 399-436.

Bonifay, E. y Vandermeersch, B. eds., (1991a): Les Premiers Européens. Actes du 114 Congrès National des Sociétès Savantes. Editions du C.T.H.C.. París.

BONIFAY, E. y VANDERMEERSCH, B., (1991b): "Vue d'ensemble sur le Très Ancien Paléolithique de l' Europe." En Les Premières Européens, E. Bonifay y B. Vandermeersch (eds.), Actes du 114 Congrès National des Sociétès Savantes. Editions du C.T.H.C.. París. pp. 309-319.

Bordes, F., (1953): "Les limons quaternaires du Bassin de la Seine." Arch. de L'l.P.H., 26. París

Bordes, F., (1971): “Observations sur l'Acheuléen des grottes en Dordogne.» Munibe, XXIII, pp. 5-23.

BORDES, F. y THIBAULT, Cl., (1977): eThougt's on the initial adaptation of hominids to european glacial climates.» Quaternary Researchs, 8, pp. 115-127.

Carbonell, E.; Bermúdez de Castro, J. M.; Arsuaga, J. L.; Diez, J. C.; Rosas, A.; CuencaBescos, G.; Sala, R.; Mosquera, M. y Rodriguez, X. P., (1995): "Lower Pleistocene Hominids and Artifacts from Atapuerca-TD6 (Spain)." Science, vol. 269, pp. 826-830.

DIAZ DEL OtMO, F. et al., (1993): "Cuaternario y secuencia paleolítica en las terrazas del Bajo y Medio Guadalquivir: aluvionamientos, coluviones, suelos y paleosuelos." En VI Jornadas de Arqueología Andaluza, Junta de Andalucia, Huelva.

FISH, P. R., (1981): "Beyond tools: Middle Paleolithic debitage analysis and cultural inference." Journal of Anthropological Research, 37, pp. 374-386.

GamBle, C. S., (1994): "Time for Boxgrove man." Nature, 369, pp. 275-276.

JELINEK, A. J., (1975): "Some current problems in Lower and Middle Paleolithic typology." Comunicación presentada a la Conference on lithic typology, Les Eyzies.

JELINEK, A. J., (1976): «Form, function, and style in lithic analysis." En Cultural change and continuity, C. E. Cleland (ed), Academic Press, Nueva York, pp. 19-34.

JONES, P. R., (1979): “Effects of raw material on biface manufacture.» Science, 204, pp. 835-836.

MARTOS, J. A., (1994): «Algunas cuestiones de interés sobre el poblamiento del continente europeo durante el Pleistoceno Medio." Espacio, Tiempo y Forma, Serie 1, Prehistoria y Arqueología, 7, pp. 13-42.

Parés, J. M. y Pérez-González, A., (1995): «Paleomagnetic Age for Hominid Fossils at Atapuerca Archaeological Site, Spain." Science, vol. 269, pp. 830-832.

Peretto, C., (1996): "Considerations on the first human population of ltaly." En Archaeology, Methodology and The Organisation of Research, S. Milliken y C. Peretto (eds) Research and excavations of the Universities and Institutes participating in the Erasmus project ICP-P. 1041, Acts of the round table, Isernia, 1994, pp. 11-17.

QUerol, M. A. y SANTonja, M., (1979): "El yacimiento achelense de Pinedo (Toledo)." Excavaciones Arqueológicas en España, 106, Madrid, Ministerio de Cultura.

Querol, M. A. y SANTonja, M., (1983): "El yacimiento de cantos trabajados de El Aculadero (Puerto de Santa María, Cádiz)." Excavaciones Arqueologicas en España, 130, Madrid, Ministerio de Cultura.

Rafoso, L. y SANTONJA, M., (1995): "The earliest occupation of Europe: the Iberian peninsula." En The earliest occupation of Europe, W. Roebroeks y T. Van Kolfschoten, (eds) 1995. Proceedings of the European Science Foundation Workshop at Tautavel (France), 1993. University of Leiden, pp. 7-25.

Robents, M. B., (1990): "Amey's Eartham Pit. Boxgrove." En C. Turner (ed), Field Excursion Guide Book, The Cromer Symposium Norwhich, 1990, SEQS, Cambridge, pp. 62-77.

Roberts, M. B.; Stringer, C. B. y Parfitt, S. A., (1994): "A hominid tibia from Middle Pleistocene sediments at Boxgrove. UK." Nature, 369, pp. 311-313.

Roche, H., (1996): «Remarques sur les plus anciennes industries en Afrique et en Europe.» En The first humans and their cultural manifestantions, F. Facchini (ed) 1996, XIII International Congress of Prehistoric and Protohistoric Sciences, Coll. VIII Lithic industries, language and social behaviour in the first human forms, Forli, Italia, pp. 55-68. 
Roebroeks, W. y Kolfschoten, T. van, (1994): "The earliest occupation of Europe: a short chronology". Antiquity, 49, pp. 489-503.

RoEBroeks, W. y KolfSCHOTEN, T. van, eds. (1995a): The earliest occupation of Europe. Proceedings of the European Science Foundation Workshop at Tautavel (France), 1993. University of Leiden.

RoEBroeks, W. y Kolfschoten, T. van, (1995b): «The earliest occupation of Europe: a reappraisal of artefactual and chronological evidence.". En The earliest occupation of Europe, $W$. Roebroeks y T. Van Kolfschote (eds.) 1995. Proceedings of the European Science Foundation Workshop at Tautavel (France), 1993. University of Leiden, pp. 297-315.

Ruiz Bustos, A. y Michaux, J., (1976): "Le site prehistorique nouveau de Cúllar-Baza 1 (Grenade, Espagne), d'age Pleistocen moyen. Étude préliminare et analyse de la faune de Rongeurs." Geologie Mediterrannée, III-3, pp. 173-182.

SANTONJA, M., (1995): "El paleolítico inferior en Europa: Apuntes en un momento de revisión." Boletín de la Asociación Española de Amigos de la Arqueología, 35, pp. 53-62.

Straus, L. G., (1980): "The role of raw material in lithic assemblages variability." Lithic technology, 9 (3), pp. 68-72.

SWISHER, C.C.; CURTIS, G. H.; JACOB, T. et al., (1994): "Age of the earliest known hominids in Java, Indonesia". Science, 263, pp. 1118-1121.

TAvoso, A., (1975): "Les hachereux sur éclats de l'Acheuléen montalbanais." Quartär, 26, pp. 13-31.

Tavoso, A., (1978): Le Paléolithique inférieur et moyen du Haut Languedoc. Tesis doctoral. Universidad de Provence, Marseille.

TURNER, A., (1995): "Variaciones regionales en la fauna de grande mamiferos del Pleistoceno Inferior y Medio en Europa." En Evolución Humana en Europa y los yacimientos de la Sierra de Atapuerca, J. L. Arsuaga y E. Carbonell (eds), pp. 57-73.

VALOCH, K., ed. (1976a): Les Premières industries de l'Europe. U.I.S.P.P., IX Congrès, Colloque VIII. Niza.

VALOCH, K., (1976b): “Aperçu des premiéres industries en Europe.» En Les Premières industries de l'Europe, K. Valoch (ed) U.I.S.P.P., IX Congrès, Colloque VIII. Niza, pp. 178-183.

VILLA, P., (1981): "Matières premières et provinces culturelles dans l'Acheulean français." Quaternaria, XXIII, Roma, pp. 19-35.

VILLA, P., (1991): «Middle Pleistocen Prehistory in Southwestern Europe: The state our knowledge and ignorance." Journal of Anthropological Research, 47 (2), pp. 193-218. 